\title{
A Bio-inspired Neural Model for Colour Image Segmentation
}

\author{
Francisco Javier Díaz-Pernas, Míriam Antón-Rodríguez, \\ José Fernando Díez-Higuera, and Mario Martínez-Zarzuela \\ Department of Signal Theory, Communications and Telematics Engineering \\ Telecommunications Engineering School, University of Valladolid, Valladolid, Spain \\ \{pacper, mirant, josdie, marmar\} @tel.uva.es
}

\begin{abstract}
This paper describes a multi-scale neural model to enhance regions and extract contours of colour-texture image taking into consideration the theory for visual information processing in the early stages of human visual system. It is composed of two main components: the Colour Opponent System (COS) and the Chromatic Segmentation System (CSS). The structure of the CSS architecture is based on BCS/FCS systems, so the proposed architecture maintains the essential qualities of the base model such as illusory contours extraction, perceptual grouping and discounting the illuminant. Experiments performed show the good visual results obtained and the robustness of the model when processing images presenting different levels of noise.
\end{abstract}

Keywords: image analysis; image segmentation; neural network; multiple scale model; Boundary Contour System; Feature Contour System; enhancing colour image regions; colour-opponent processes.

\section{Introduction}

Image segmentation is a difficult yet very important task in many image analysis or computer vision applications. Segmentation subdivides an image into its constituent parts or object. It depends on the global characteristics of an image, which is similar to the judgment of human perception. The proposed model covers the processing of the segmentation stage in a biological way, processing colour and textural information for intensifying regions and extracting perceptual boundaries as a previous processing, prior to region labelling, in order to form up the segmented image. This image enhancing makes labelling easier during the segmentation process and also makes it more efficient by reducing the uncertainty of the images' region allocation.

The skill of identifying, grouping and distinguishing among textures and colours is inherent to the human visual system. For the last few years many techniques and models have been proposed in the area of textures and colour analysis [5], resulting in a detailed characterisation of both parameters. Many of these initiatives, however, have used geometric models, omitting the human vision physiologic base. The main difference, and advantage, of the human visual system is the context dependence [8]; this implies that its processing cannot be simulated by means of geometric techniques 
that obviate this information. A clear example of such a feature is the illusory contour formation, in which context data is used to complete the received information, which is partial or incomplete in many cases.

The architecture described in this work is based on the BCS/FCS neural model, composed of the Boundary Contour System and the Feature Contour System introduced by [7] and [3]. This model suggests a neural dynamics for perceptual segmentation of monochromatic visual stimuli and offers a unified analysis process for different data referring to monocular perception, grouping, textural segmentation and illusory figures perception. The BCS system obtains a map of image contours based on contrast detection processes, whereas the FCS performs diffusion processes with luminance filling-in within those regions limited by high contour activities. Consequently, regions that show certain homogeneity and are globally independent are intensified. Later versions of BCS/FCS models, [9] [15] have considered multiscale processing, in which the system is sensitive to boundaries and surfaces of different sizes. Recent publications within the Theory of Neural Dynamics of the Visual Perception focus on the modelling of retinal processes in order to solve the problem of consistent interpretation of surface lightness under varying illumination.

In this manner, the main improvement introduced to the original model hereby in this paper, resides in offering a complete colour image processing neural architecture for extracting contours and enhancing the homogeneous areas in an image. In order to do this, the neural architecture develops processing stages, coming from the original RGB image up to the segmentation level, following analogous behaviours to those of the early mammalian visual system. This adaptation has been performed by trying to preserve the original BCS/FCS model structure and its qualities, establishing a parallelism among different visual information channels and modelling physiological behaviours of the visual system processes. Therefore, the region enhancement is based on the feature extraction and perceptual grouping of region points with similar and distinctive values of luminance, colour, texture and shading information.

The paper is organized as follows. In Section 2, we review the segmentation algorithm. Section 3 studies its performance over input images presenting different noise levels; and finally a conclusion is drawn in Section 4.

\section{Proposed Neural Model}

The architecture of the proposed model (Fig. 1) comprises of two main components, designated respectively Colour Opponent System (COS) and Chromatic Segmentation System (CSS).

The COS module transforms the chromatic components of the input signals (RGB) into a bio-inspired codification system, made up of two opponent chromatic channels, L-M and S-(L+M), and an achromatic channel. Resulting signals from COS are used as inputs for the CSS module where the contour map extraction and two intensified region images, corresponding to the enhancement of $\mathrm{L}-\mathrm{M}$ and $\mathrm{S}-(\mathrm{L}+\mathrm{M})$ opponent chromatic channels, are generated in multiple scale processing.

The final output is constituted by three components: an image contour map and two intensified region images corresponding to the enhancement of aforementioned L-M and S-(L+M) opponent chromatic channels. 


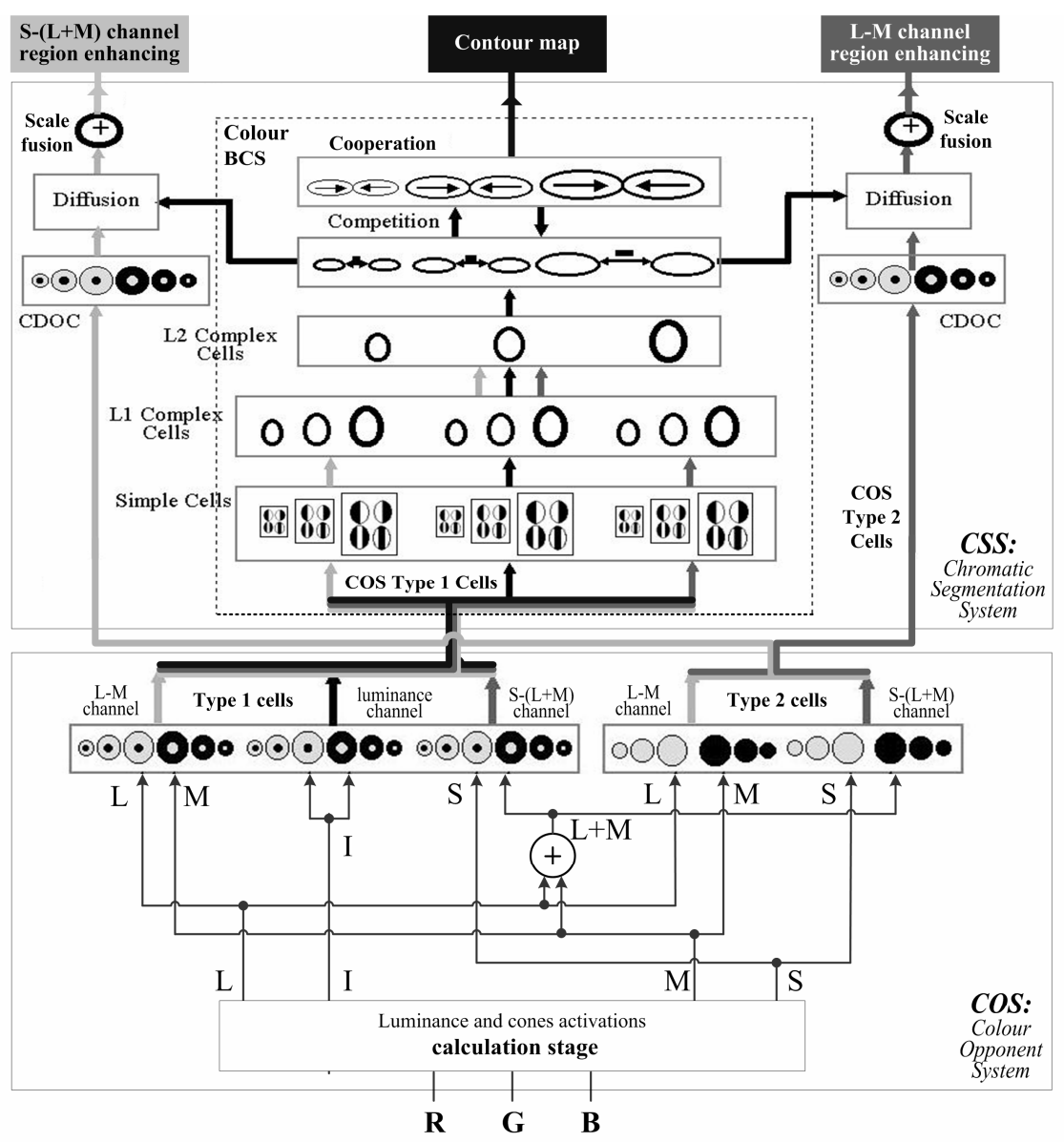

Fig. 1. Proposed model architecture. At the bottom, the detailed COS module structure: on the left, it shows type 1 cells whereas on the right, elements correspond to type 2 opponent cells. At the top, the detailed structure of the CSS based on the BCS/FCS model.

\subsection{Colour Opponent System (COS)}

The COS module performs colour opponent processes based on opponent mechanisms that are present on the retina and on the LGN of the mammalian visual system [10] [17]. Firstly, luminance (I signal) and activations of the long (L), middle $(\mathrm{M})$, short $(\mathrm{S})$ wavelength cones and $(\mathrm{L}+\mathrm{M})$ channel activation ( $\mathrm{Y}$ signal) are generated from R, G and B input signals. The I signal is computed as a weighted sum [5]; the L, M and S signals are obtained as the transformation matrix [11].

In the COS stage, two kinds of cells are suggested; type 1 and type 2 cells (see Fig. 1). These follow opponent profiles intended for detecting contours (type 1, simple opponency) and colour diffusion (type 2 cells initiate double opponent processes). 


\section{Type 1 Opponent Cells}

Type 1 opponent cells perform a colour codification system represented by the existence of opponent L-M, S-(L+M), and luminance channels (see Fig. 1). These cells are modelled through two centre-surround multiple scale competitive networks, and form the ON and OFF channels composed of ON-centre OFF-surround and OFFcentre ON-surround competitive fields, respectively. These competitive processes establish a gain control network over the inputs from chromatic and luminance channels, maintaining the sensibility of cells to contrasts, compensating variable illumination, and normalising image intensity [6] [8]. The equations governing the activation of type 1 cells (1) have been taken from the Contrast Enhancement Stage in [9] [15], but adapted to compute colour images.

$$
\begin{array}{ccc}
y_{i j}^{g+}=\left[\frac{A D^{+}+B S_{i j}^{c}-C S_{i j}^{s g}}{A+S_{i j}^{c}+S_{i j}^{s g}}\right]^{+} ; & y_{i j}^{g-}=\left[\frac{A D^{-}+C S_{i j}^{s g}-B S_{i j}^{c}}{A+S_{i j}^{s g}+S_{i j}^{c}}\right]^{+} \\
S_{i j}^{c}=\sum_{p q} e_{i+p, j+q}^{c} G_{p q}^{c} ; & S_{i j}^{s g}=\sum_{p q} e_{i+p, j+q}^{s g} G_{p q}^{s g}
\end{array}
$$

with $A, B, C$ and $D$ as model parameters, $[w]^{+}=\max (w, 0), e^{c}$ as central signal, $e^{s}$ as peripheral signal (see Table 1), the superscript $g=0,1,2$ for small, medium and large scales. The weight functions have been defined as normalised Gaussian functions for central $\left(G^{c}\right)$ and peripheral $\left(G^{s g}\right)$ connectivity.

Table 1. Inputs of different channels on type 1 opponent cells

\begin{tabular}{cccc}
\hline & L-M Opponency & S-(L+M) Opponency & Luminance \\
\hline $\mathrm{e}^{\mathrm{c}}$ & $\mathrm{L}_{\mathrm{ij}}$ & $\mathrm{S}_{\mathrm{ij}}$ & $\mathrm{I}_{\mathrm{ij}}$ \\
$\mathrm{e}^{\mathrm{sg}}$ & $\mathrm{M}_{\mathrm{ij}}$ & $\mathrm{Y}_{\mathrm{ij}}$ & $\mathrm{I}_{\mathrm{ij}}$ \\
\hline
\end{tabular}

\section{Type 2 Opponent Cells}

The type 2 opponent cells initiate the double opponent process that take place in superior level, chromatic diffusion stages (see Fig. 1). The double opponent mechanisms are fundamental in human visual colour processing [10].

The receptive fields of type 2 cells are composed of a unique Gaussian profile. Two opponent colour processes occur, corresponding L-M and $\mathrm{S}-(\mathrm{L}+\mathrm{M})$ channels (see Fig. 1). Each opponent process is modelled by a multiplicative competitive central field, presenting simultaneously an excitation and an inhibition caused by different types of cone signals ( $\mathrm{L}, \mathrm{M}, \mathrm{S}$ and $\mathrm{Y}$ as sum of $\mathrm{L}$ and $\mathrm{M}$ ). These processes are applied over three different spatial scales in the multiple scale model shown. Equations (3) model the behaviour of these cells, ON and OFF channels, respectively.

$$
x_{i j}^{g+}=\left[\frac{A D^{+}+B S_{i j}^{+g}}{A+S_{i j}^{\mathrm{Eg}}}\right]^{+} ; \quad x_{i j}^{g-}=\left[\frac{A D^{-}+B S_{i j}^{-g}}{A+S_{i j}^{\mathrm{Eg}}}\right]^{+}
$$




$$
\begin{gathered}
S_{i j}^{+g}=\sum_{p q} G_{p q}^{g}\left(e_{i+p, j+q}^{(1)}-e_{i+p, j+q}^{(2)}\right) ; S_{i j}^{-g}=\sum_{p q} G_{p q}^{g}\left(e_{i+p, j+q}^{(2)}-e_{i+p, j+q}^{(1)}\right) \\
S_{i j}^{\mathrm{Eg}}=\sum_{p q} G_{p q}^{g}\left(e_{i+p, j+q}^{(1)}+e_{i+p, j+q}^{(2)}\right)
\end{gathered}
$$

with $A, B, C$ and $D$ as model parameters, $[w]^{+}=\max (w, 0), e^{(1)}$ and $e^{(2)}$ being the input signals of the opponent process (see Table 2). The weight functions have been defined as normalised Gaussians with different central connectivity $\left(G^{g}\right)$ for the different spatial scales $\mathrm{g}=0,1,2$ (see Table 4 ).

Table 2. Inputs for different type 2 cells channels

\begin{tabular}{ccc}
\hline & L-M Opponency & S-(L+M) Opponency \\
\hline $\mathrm{e}^{(1)}$ & $\mathrm{L}_{\mathrm{ij}}$ & $\mathrm{S}_{\mathrm{ij}}$ \\
$\mathrm{e}^{(2)}$ & $\mathrm{M}_{\mathrm{ij}}$ & $\mathrm{Y}_{\mathrm{ij}}$ \\
\hline
\end{tabular}

\subsection{Chromatic Segmentation System (CSS)}

As previously mentioned, the Chromatic Segmentation System bases its structure on the modified BCS/FCS model [9] [15], adapting its functionality for colour image processing. The CSS module (see Fig. 1) consists of the Colour BCS stage and two chromatic diffusion stages, processing one chromatic channel each.

\section{Colour BCS stage}

The Colour BCS stage constitutes our colour extension of the original BCS model. It processes visual information from three parallel channels, two chromatic and a luminance channels to obtain a unified contour map. Analogous to the original model, the Colour BCS module has two differentiated phases: the first one (simple and complex cells) extracts real contours from the output signals of the COS and the second is represented by a competition and cooperation loop, in which real contours are completed and refined, thus generating contour interpolation and illusory contours (see Fig. 1). Colour BCS preserves all of the original model perceptual characteristics such as perceptual grouping, emergent features and illusory perception.

The achieved output coming from the competition stage is a contour map of the original image. This output is transmitted to the diffusion stages where it will act as a control signal serving as a barrier in chromatic diffusions.

Simple cells are in charge of extracting real contours from each of the chromatic and luminance channels. In this stage, the filters from the original model have been replaced by two pairs of Gabor filters with opposite polarity, due to their high sensibility to orientation, spatial frequency and position [4] [13]. Their presence has been proved on the simple cells situated at V1 area of visual cortex [16]. Fig. 2 shows a visual representation of Gabor filter pair profiles. 


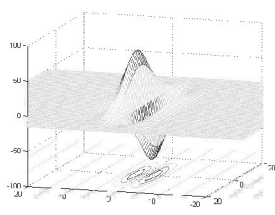

a)

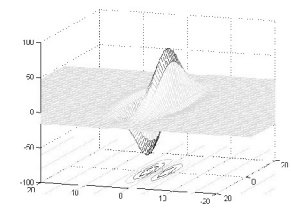

b)

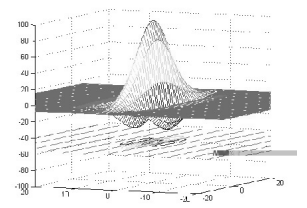

c)

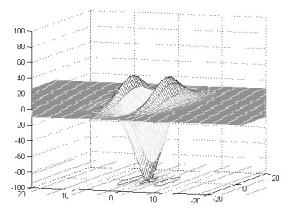

d)

Fig. 2. Receptive fields of the filters used to model simple cells. a) Anti-symmetric light-dark receptive field. b) Anti-symmetric dark-light receptive field. c) Symmetric receptive field with central excitation. d) Symmetric receptive field with central inhibition.

The complex cell stage, using two cellular layers, fuses information from simple cells giving rise to a map which contains real contours for each of the three scales used (see Fig. 1). Detected real contours are passed into a cooperative-competitive loop. This nonlinear feedback network detects, regulates, and completes boundaries into globally consistent contrast positions and orientations, while it suppresses activations from redundant and less important contours, thus eliminating image noise. The loop completes the real contours in a consistent way generating, as a result, the illusory contours [9] [15].

Cooperation is carried out by dipole cells, which have been placed just before cortical cells in V2 area. These cells have been used to model processes such as illusory contour generation, neon colour spreading or texture segregation [7]. Dipole cells act like long-range statistical AND gates, providing active responses if they perceive enough activity over both dipole receptive fields lobes (left and right). Thus, this module performs a long-range orientation-dependent cooperation in such a way that dipole cells are excited by collinear (or close to collinearity) competition outputs and inhibited by perpendicularly oriented cells. This property is known as spatial impermeability and prevents boundary completions towards regions containing substantial amounts of perpendicular or oblique contours. The equations used in competitive and cooperative stages are taken from the original model [9].

\section{Chromatic diffusion stages}

As mentioned above, the chromatic diffusion stage has undergone changes that entailed the introduction of Chromatic Double Opponency Cells (CDOC), resulting in a new stage in the segmentation process.

In human visual system, double opponency occurs in visual striate cortex cells, contained in blobs [10]. The model for these cells has the same receptive field as COS type 1 opponent cells (centre-surround competition), but their behaviour is quite a lot more complex since they are highly sensitive to chromatic contrasts. Double opponent cell receptive fields are excited on their central region by COS type 2 opponent cells, and are inhibited by the same cell type. We apply a greater sensibility to contrast as well as a more correct attenuation toward illumination effects, therefore bringing a positive solution to the noise-saturation dilemma.

The mathematical pattern that governs the behaviour of CDOC cells is the one defined by (1) and successive equations, by varying only their inputs, that is, the outputs of the COS type 2 opponent cells for each chromatic channel (see Table 3 ). 
Table 3. Inputs of included Chromatic Double Opponency Cells

\begin{tabular}{lllll}
\hline & \multicolumn{2}{c}{ L-M Opponency } & \multicolumn{2}{c}{ S-(L+M) Opponency } \\
\hline $\mathrm{e}^{\mathrm{c}}$ & $\left(\mathrm{L}^{+}-\mathrm{M}^{-}\right)_{\mathrm{ij}}$ & $\left(\mathrm{L}^{-}-\mathrm{M}^{+}\right)_{\mathrm{ij}}$ & $\left(\mathrm{S}^{+}-\mathrm{Y}^{-}\right)_{\mathrm{ij}}$ & $\left(\mathrm{S}^{-}-\mathrm{Y}^{+}\right)_{\mathrm{ij}}$ \\
$\mathrm{e}^{\mathrm{sg}}$ & $\left(\mathrm{L}^{+}-\mathrm{M}^{-}\right)_{\mathrm{ij}}$ & $\left(\mathrm{L}^{-}-\mathrm{M}^{+}\right)_{\mathrm{ij}}$ & $\left(\mathrm{S}^{+}-\mathrm{Y}^{-}\right)_{\mathrm{ij}}$ & $\left(\mathrm{S}^{-}-\mathrm{Y}^{+}\right)_{\mathrm{ij}}$ \\
\hline
\end{tabular}

Chromatic diffusion stages perform four nonlinear and independent diffusions for L-M (ON and OFF) and S-Y (ON and OFF) chromatic channels. These diffusions are controlled by means of a final contour map obtained from the competitioncooperation loop while the outputs of CDOC are the signals being diffused. At this stage, each spatial position diffuses its chromatic features in all directions except those in which a boundary is detected. When boundary signals take part, they inhibit diffusion obtaining differentiated activities at each of their sides (thus separating regions with different features) [15]. By means of this process, image regions that are surrounded by closed boundaries tend to obtain uniform chromatic features, even in noise presence, and therefore producing the enhancement of the regions detected in the image. The equations that model the diffusive filling-in can be found in [9].

Scale fusion constitutes the last stage of this pre-processing architecture. A simple linear combination of the three scales (6), obtains suitable visual results at this point.

$$
V_{i j}=A_{0}\left(F_{i j}^{01}-F_{i j}^{02}\right)+A_{1}\left(F_{i j}^{11}-F_{i j}^{12}\right)+A_{2}\left(F_{i j}^{21}-F_{i j}^{22}\right)
$$

where $A_{0}, A_{1}$ and $A_{2}$ are linear combination parameters, $F_{i j}^{g t}$ represents diffusion outputs, with $g$ indicating the spatial scale $(g=0,1,2)$ and $t$ denoting the diffused double opponent cell, 1 for $\mathrm{ON}$ and 2 for OFF.

\section{Tests and Results}

This section introduces our tests' simulations over the proposed architecture. Selected colour images are shown in Fig. 3. The format of the images is RGB, with 24 bits per pixel. Each of them shows diverse features that, altogether, validate the model in a general way and also against changing requirements (scale, chromatic, texture...).
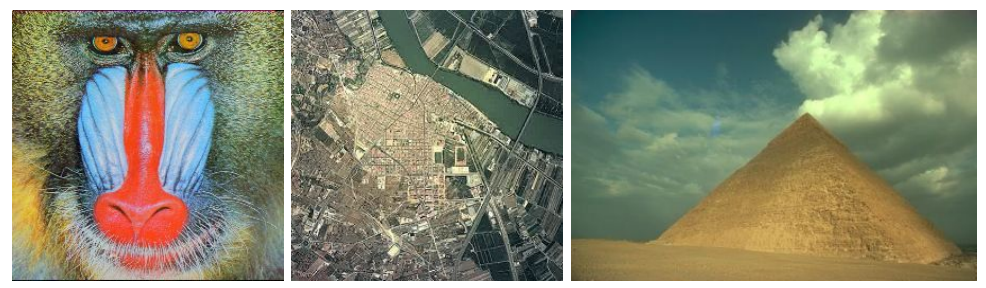

Fig. 3. Colour images included in the tests: Mandrill, Aerial, and Pyramid

The offset model parameters were adjusted to obtain the best visual results (see Table 4). 
Table 4. Parameter values of the proposed architecture for test simulations

\begin{tabular}{|c|c|c|c|}
\hline \multicolumn{2}{|c|}{$\begin{array}{c}\text { Type } 1 \text { COS cells } \\
\text { L-M, S-(L+M) and Luminance Opponencies }\end{array}$} & \multicolumn{2}{|c|}{$\begin{array}{c}\text { Type } 2 \text { COS cells } \\
\text { L-M and } \mathrm{S}-(\mathrm{L}+\mathrm{M}) \text { Opponencies }\end{array}$} \\
\hline A 1000.0 & $\sigma_{\mathrm{c}} 0.3$ & A 10000.0 & $\sigma_{\mathrm{s}} 0.3$ \\
\hline C 1.0 & $\sigma_{\mathrm{ss}} \quad 0.5$ & В 10.0 & $\sigma_{\mathrm{m}} \quad 0.8$ \\
\hline B 1.0 & $\sigma_{\mathrm{sm}} 1.0$ & $\mathrm{D}^{+} \quad 10.0$ & $\sigma_{1} 1.6$ \\
\hline $\mathrm{D}^{+}, \mathrm{D}^{-} \quad 1.0$ & $\sigma_{\mathrm{sl}} 1.8$ & $\mathrm{D}^{-} \quad 10.0$ & \\
\hline \multicolumn{2}{|c|}{$\begin{array}{c}\text { Simple cells } \\
\text { L-M, S-(L+M) and Luminance Opponencies }\end{array}$} & \multicolumn{2}{|c|}{$\begin{array}{c}\text { Complex cells } \\
\mathrm{M}, \mathrm{S}-(\mathrm{L}+\mathrm{M}) \text { and Luminance Opponencies }\end{array}$} \\
\hline $\mathrm{F}_{\mathrm{s}} 12.0$ & $\sigma_{\mathrm{ss}} 8.0$ & $\gamma \quad 1.0$ & $\xi 0.01$ \\
\hline $\mathrm{F}_{\mathrm{m}} 8.0$ & $\sigma_{\mathrm{sm}} 12.0$ & $\kappa 1.0$ & v 0.01 \\
\hline $\mathrm{F}_{1} \quad 5.0$ & $\sigma_{\mathrm{sl}} \quad 15.0$ & & \\
\hline \multicolumn{2}{|c|}{ Competition cells } & \multicolumn{2}{|c|}{ Cooperation cells } \\
\hline A 3.0 & $\sigma_{\mathrm{s}} 4.0$ & A 3.0 & $\mathrm{C}_{\mathrm{ws}}, \mathrm{C}_{\mathrm{wm}}, 40$ \\
\hline B 1.0 & $\sigma_{\mathrm{m}} 8.0$ & B 1.0 & $\mathrm{C}_{\mathrm{wl}} 4.0$ \\
\hline C 0.2 & $\sigma_{1} 16.0$ & $\mathrm{C}_{\mathrm{ls}} \quad 0.2$ & T 2.0 \\
\hline $\mathrm{G}_{\mathrm{f}} \quad 1.0$ & $\sigma_{\mathrm{k}} 45.0$ & $\mathrm{C}_{\operatorname{lm}} 1.0$ & $\mu \quad 11.0$ \\
\hline $\mathrm{G}_{\mathrm{b}} \quad 15.0$ & & $\mathrm{C}_{\mathrm{ll}} \quad 15.0$ & $\beta 0.8$ \\
\hline \multicolumn{2}{|c|}{$\begin{array}{c}\text { CDOC cells } \\
\mathrm{L}-\mathrm{M} \text { and } \mathrm{S}-(\mathrm{L}+\mathrm{M}) \text { Opponencies }\end{array}$} & Diffusion stage & Scale fusion \\
\hline A 10000.0 & $\sigma_{\mathrm{ss}} 1.2$ & D 150.0 & $\mathrm{~A}_{0} \quad 8.0$ \\
\hline $\mathrm{D}^{+} \quad 1.0$ & $\sigma_{\mathrm{sm}} 5.2$ & $\delta \quad 20000.0$ & $\mathrm{~A}_{1} \quad 2.0$ \\
\hline $\mathrm{D}^{-} 1.0$ & $\sigma_{\mathrm{sl}} 10.8$ & $\varepsilon \quad 30.0$ & $\mathrm{~A}_{2} \quad 1.0$ \\
\hline$\sigma_{\mathrm{c}} \quad 0.3$ & $\sigma_{\mathrm{ss}} \quad 1.2$ & & \\
\hline
\end{tabular}
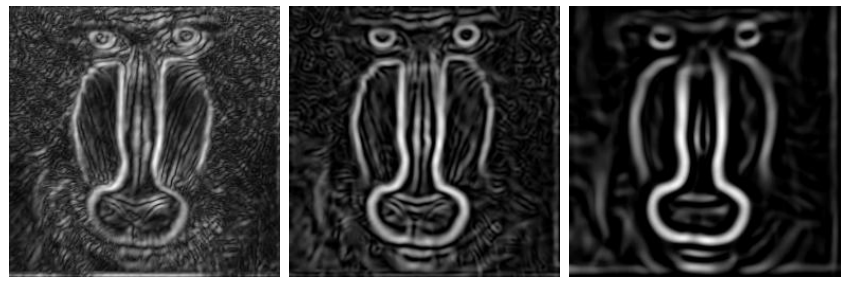

Fig. 4. Final boundary maps at three spatial scales, obtained from the competition stage

In Fig. 4, the final boundary maps obtained from the Colour BCS module are depicted. Small scale performs higher precision and contrast sensitivity even though it presents higher noise level (e.g. mandrill hair), while large scale obtains lesser precision and contrast sensitivity but also higher noise filtering. The BCS colour module has extracted the mandrill's nose contour in a continuous way. This is due to the interaction among the competition and cooperation processes which generate mechanisms of contour interpolation and illusory contour extraction.

In order to evaluate the contour extraction capabilities of the described model, we compared it to the well known Canny extractor [1], using the cvCanny() function from Intel Computer Vision Library, OpenCv [12]. Parameters were assigned using best visual result criteria. For mandrill's image we use: threshold $1=2000$, 

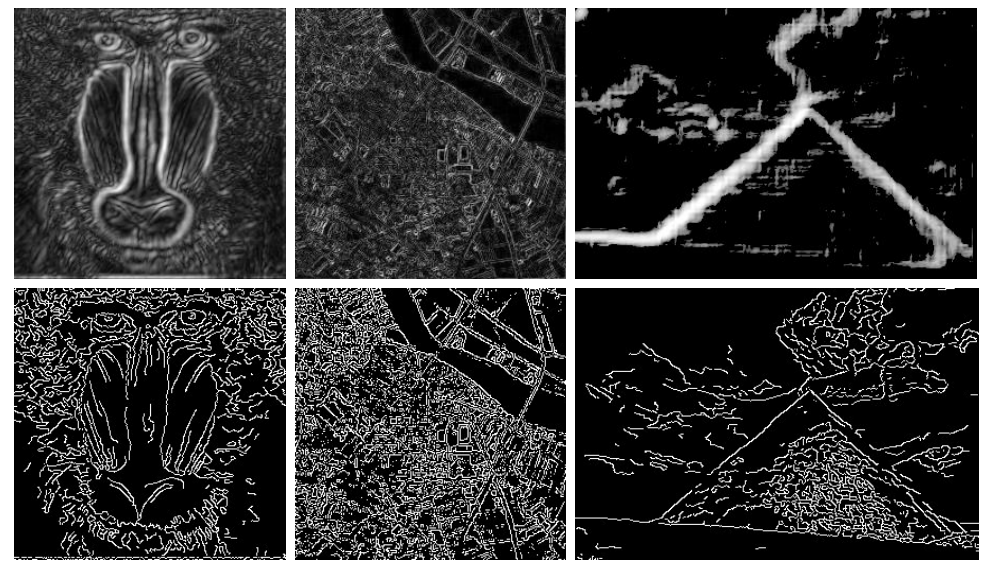

Fig. 5. Comparison with Canny's contour extraction. Top row: Our model, Bottom row: Canny.

threshold $2=1000$, and aperture size $=5$; for Aerial image: $\mathrm{t} 1=200, \mathrm{t} 2=200$, and $\mathrm{a}=3$; and for Pyramid image: $\mathrm{t} 1=10000, \mathrm{t} 2=5000$, and $\mathrm{a}=7$.

In Fig. 5, top row shows the contour's structure of our model while bottom row shows the output of the Canny extractor. It can be note that Canny's could not extract the mandrill's nose as a continuous contour, unlike our model. The Aerial image processing shows how the model responds to very detailed images with fine contours, demonstrating a great level of precision at small scales. Finally, the Pyramid image allows us to observe the behaviour of the model when processing large scale, obtaining well defined contours and achieving a strong elimination of the noise, which exists in Canny extraction.

Fig. 6 depicts the final outputs of the model after fusing information from the diffusion stage with the three spatial scales (see Table 4). As it can be noted, chromatic and textural features appear now levelled within each enhanced region. Our
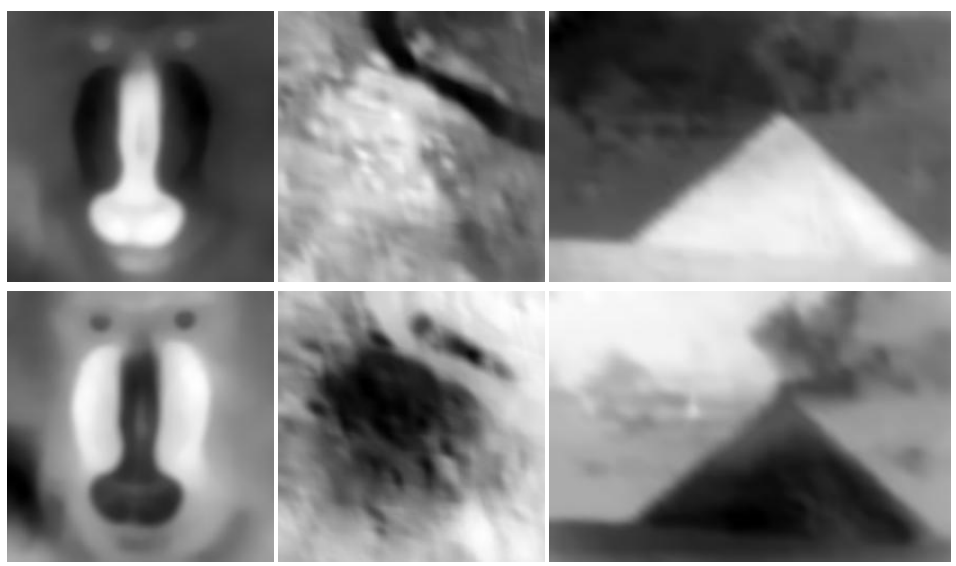

Fig. 6. Final outputs of the model. Top row: L-M channel. Bottom row: S-Y channel. 

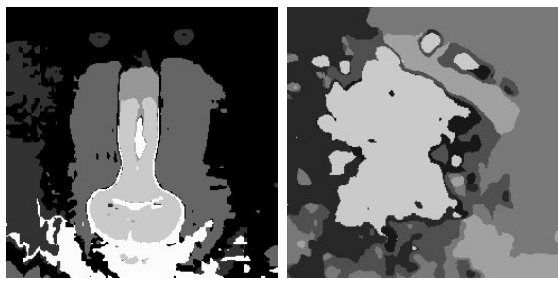

Fig. 7. Labelling of the featured model's output using a Fuzzy ART Network. Supervision parameter $(\rho)$ is 0.9. Left: Mandrill, 7 categories/regions. Right: Aerial, 9 categories/regions.

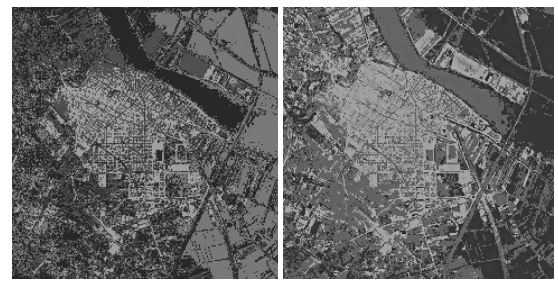

Fig. 8. Comparison with other segmentation schemas. Left: Original RGB image labelling. Right: Results from the pyramidal segmentation.

model has enhanced the homogeneous regions of Mandrill and Aerial images. The enhancement could be used to estimate towns or places' population from aerial images. The Pyramid image, however, contains a heterogeneous sky with clouds which are distinguished as different enhanced regions by the model. This image also obtains a good visual result from a textured region such as the pyramid's surface.

In order to obtain segmentation, after enhancing regions of the image, the immediate following process will be the region labelling, that is, making all the points belonging the same region have the same region label. In fact, enhancing regions makes the labelling process easier and so more efficient. So as to prove this statement, a Fuzzy ART categorisation model [2] was chosen to run the labelling process. For each point in the image, a four component pattern, composed of the output values from chromatic diffusion stages and their complement coding, is taken, thus the segmentation image is generated by labelling all of the points in accordance with their pattern category, giving a different grey level to each category for result visualization. Fig. 7 shows the categorisation for the Mandrill and Aerial images. In both cases the Fuzzy ART supervision value $\rho$ was $0.9 ; 7$ and 9 categories were created respectively.

To show how the results coming from our architecture favour the segmentation process in comparison with other segmentation models, we used the Aerial image from Fig. 3. Fig. 8-left shows the RGB original image labelling using the same Fuzzy ART model, with the same value for the supervision parameter $(\rho=0.9)$. This categorisation created 10 categories. Aerial image was also segmented through a pyramidal process (Fig. 8-right) using the cvPyrSegmentation() function from the OpenCv library [12]. The parameters' values were assigned using the best visual results criterion $(\mathrm{t} 1=70, \mathrm{t} 2=30)$. By comparing Fig. 7-right with Fig. 8, it can be easily observed that the introduced model takes into account the colour and textural 

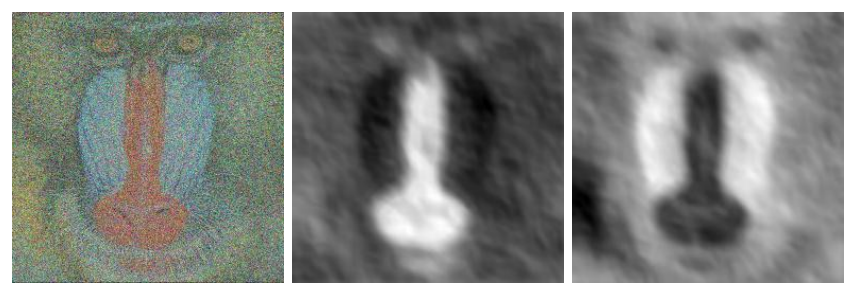

Fig. 9. Results for image with $30 \%$ of additive Gaussian noise on each channel. It can be seen that the enhancement results are visually satisfactory even with a high level of noise.

information and this is the reason why it detects a wide central region, in a compatible way with human visual perception, unlike the two other methods. Hence, it can be inferred that the presented architecture obtains far more satisfactory results than the pyramidal method.

In order to validate the architecture against adverse circumstances, some Gaussian noise has been added to each of the R, G, and B input channels. This kind of noise is usually introduced by acquisition devices on industrial applications. Consequently, noise is individually measured as a percentage of noise power over the input channel total power (noise + signal). When additive Gaussian noise is applied to input images, the system responses are satisfying up to the point when a $30 \%$ noise level is reached on each channel. As shown in Fig. 9, obtained segmentation give good visual results although detected regions are not as homogeneous as with clean images, they appear notoriously defined. It is important to stress the better performance at larger scales when noise increases, as their weight becomes higher on fusion stages.

\section{Conclusion}

This work presents a multiple scale neural model for extracting contours and enhancing regions in colour images. The model processes chromatic information to obtain a coherent region enhancing. BCS/FCS systems have been used as a baseline due to their broad validation and acceptance. Furthermore, these systems have been extended with the addition of the stages performing chromatic processing. The use of a multiple scale model has demonstrated a great level of flexibility and adaptation to multiple images, which lays the foundations to obtain a more general purpose architecture for colour image segmentation.

Although it increases the system's complexity and the computational load (more parallel processing and more stages are added), colour processing through type 1 and 2 COS cells and CDOC cells provides significant improvements on the segmentation and discriminates better among regions with similar luminance values but different chromatic features. Furthermore, the architecture shows a great level of parallelism and therefore computation load can be shared among different processors.

We included test simulations in order to validate the model, obtaining really satisfactory visual results. When comparing it to Canny's contour extractor we have observed that the perceptual contour extraction used by our model displays features not present in Canny's like, for example, illusory contours detection and perceptual grouping which helps us to obtain results much more in line with those of human 
visual perception. The results shown have covered different areas in which computer vision systems have become a helpful solution and a means of automating tools such as face recognition systems or aerial images analysis.

Finally, this paper demonstrates that the described architecture displays a visually satisfactory response against the standard Gaussian noise present in most image acquisition devices.

\section{References}

1. Canny, J.: A computational approach to edge detection. IEEE Trans. Pat. Anal. Mach. Intell. 8(6), 679-698 (1986)

2. Carpenter, G.A., Grossberg, S., Rosen, D.B.: Fuzzy ART: Fast Stable Learning and Categorization of Analog Patterns by an Adaptive Resonance System. Neural Networks 4, 759-771 (1991)

3. Cohen, M.A., Grossberg, S.: Neural dynamics of brightness perception: features, boundaries, diffusion, and resonance. Perception and Psychophysics 36, 428-456 (1984)

4. Daugman, J.G.: Two-dimensional spectral analysis of cortical receptive field profiles. Vision Research 20, 847-856 (1980)

5. Gonzalez, R.C., Woods, R.E.: Digital Image Processing, 2/E. Prentice Hall, Englewood Cliffs (2002)

6. Grossberg, S.: Contour enhancement, short term memory, and constancies in reverberating neural networks. Studies in Applied Mathematics 52, 217-257 (1973)

7. Grossberg, S.: Outline of a theory of brightness, colour, and form perception. In: Degreef, E., van Buggenhault, J. (eds.) Trends in mathematical psychology. North Holland, Amsterdam (1984)

8. Grossberg, S., Mingolla, E.: Neural dynamics of perceptual grouping: textures, boundaries, and emergent segmentations. In: Grossberg, S. (ed.) The adaptive brain II, ch. 3. North Holland, Amsterdam (1988)

9. Grossberg, S., Mingolla, E., Williamson, J.: Synthethic aperture radar processing by a multiple scale neural system for boundary and surface representation. Neural Networks 8 , 1005-1028 (1995)

10. Hubel, D.H.: Eye, Brain and Vision. Scientific American Library 22, 70 (1995)

11. Hubel, D.H., Livingstone, M.S.: Color and contrast sensitivity in lateral geniculate body and Primary Visual Cortex of the Macaque Monkey. The Journal of Neuroscience 10(7), 2223-2237 (1990)

12. Intel Corporation, Open Source Computer Vision Library (2006), http: / / www. intel.com/technology/computing/opencv/

13. Landy, M.S., Bergen, J.R.: Texture segregation and orientation gradient. Vision Research 31(4), 679-693 (1991)

14. Mirmehdi, M., Petrou, M.: Segmentation of color textures. IEEE Trans. Pattern Analysis and Machine Intelligence 22(2), 142-159 (2000)

15. Mingolla, E., Ross, W., Grossberg, S.: A neural network for enhancing boundaries and surfaces in synthetic aperture radar images. Neural Networks 12, 499-511 (1999)

16. Pollen, D.A., Ronner, S.F.: Visual cortical neurons as localized spatial frequency filters. IEEE Transactions on Systems, Man, and Cybernetics SMC-13(15), 907-916 (1983)

17. Wilson, H.R., Levi, D., Maffei, L., Rovamo, J., De Valois, R.: The Perception of Form: Retina to Striate Cortex. In: En Spillmann, L., Werner, J.S. (eds.) Visual Perception: The Neurophysiological Foundations, ch.10. Academic Press, San Diego (1990) 